\title{
Glycyrrhizin attenuates endotoxin- induced acute liver injury after partial hepatectomy in rats
}

B. Tang ${ }^{1}$, H. Qiao ${ }^{1}$, F. Meng ${ }^{2}$ and X. Sun ${ }^{1}$

\author{
1The Hepatosplenic Surgery Center/Department of General Surgery, \\ The First Clinical Medical School, Harbin Medical University, \\ Harbin, Heilongjiang Province, China \\ ${ }^{2}$ The 2nd Department of General Surgery, China-Japan Friendship Hospital, \\ Beijing, China
}

\section{Correspondence \\ X. Sun \\ The Hepatosplenic Surgery Center Department of General Surgery The First Clinical Medical School Harbin Medical University 23 Youzheng Street Harbin, Heilongjiang Province 150001 China \\ Fax: +86-451-5364-3628 \\ E-mail: kevsun88@hotmail.com \\ Research supported by the National Natural Scientific Foundation of China (Nos. 30571753, 30471681, and 30571808), a grant from the Creative Foundation for postgraduates at Harbin Medical University (No. 200401087) to B. Tang, and a Collaborative Fund for Oversea Scholars from the Science and Technology Bureau of Heilongjiang Province, China (WH05C02).}

Received December 8, 2006 Accepted July 16, 2007

\begin{abstract}
Massive hepatectomy associated with infection induces liver dysfunction, or even multiple organ failure and death. Glycyrrhizin has been shown to exhibit anti-oxidant and anti-inflammatory activities. The aim of the present study was to investigate whether glycyrrhizin could attenuate endotoxin-induced acute liver injury after partial hepatectomy. Male Wistar rats (6 to 8 weeks old, weighing 200-250 g) were randomly assigned to three groups of 24 rats each: sham, saline and glycyrrhizin. Rats were injected intravenously with lipopolysaccharide (LPS) $24 \mathrm{~h}$ after $70 \%$ hepatectomy. Glycyrrhizin, pre-administered three times with $24 \mathrm{~h}$ intervals $48 \mathrm{~h}$ before hepatectomy, prolonged the survival of rats submitted to partial hepatectomy and LPS injection, compared with saline controls. Glycyrrhizin was shown to attenuate histological hepatic changes and significantly reduced serum levels of aspartate aminotransferase, alanine aminotransferase, and lactic dehydrogenase, at all the indicated times (6 rats from each were sacrificed 1, 3, 6, and $9 \mathrm{~h}$ after LPS injection), compared with saline controls. Glycyrrhizin also significantly inhibited hepatocyte apoptosis by down-regulating the expression of caspase- 3 and inhibiting the release of cytochrome $\mathrm{C}$ from mitochondria into the cytoplasm. The anti-inflammatory activity of glycyrrhizin may rely on the inhibition of release of tumor necrosis factor- $\alpha$, myeloperoxidase activity, and translocation of nuclear factor-kappa B into the nuclei. Glycyrrhizin also up-regulated the expression of proliferating cell nuclear antigen, implying that it might be able to promote regeneration of livers harmed by LPS. In summary, glycyrrhizin may represent a potent drug protecting the liver against endotoxin-induced injury, especially after massive hepatectomy.
\end{abstract}

Extensive hepatectomy is common for hepatic malignancies. Despite improvement in surgical techniques and perioperative management, liver failure remains one of the

\section{Introduction}

Key words

- Glycyrrhizin

- Lipopolysaccharide

- Hepatectomy

- Inflammatory cytokine

- Apoptosis

- Endotoxemia 
cal formation (4), inflammatory mediators $(5,6)$ such as tumor necrosis factor-alpha (TNF- $\alpha$ ), interleukin-1ß, and cytokine-induced neutrophil chemoattractant, and activation of the transcription factor, nuclear factor-kappa B (NF- $\mathrm{B}$ ) (7). Glycyrrhizin, a natural compound extracted from the roots of Glycyrrhiza glabra, has been used for more than two decades to treat hepatitis in Japan, and no side effect or toxicity has been observed (8). Its major constituents are glycyrrhetic acid, multiple flavonoids, isoflavonoids, hydroxycoumarins, and sterols (9). Glycyrrhizin inhibits CD4+ $\mathrm{T}$ cell- and TNF-mediated cytotoxicity (10), and has a membrane-stabilizing effect (11). Because of its anti-inflammatory and antioxidant activities (9), glycyrrhizin has been shown to protect the liver (12) and kidneys (13) from ischemia-reperfusion injury in animal models. Therefore, we designed this study to determine whether glycyrrhizin protects against acute liver injury induced by endotoxemia after massive hepatectomy.

\section{Material and Methods}

\section{Animals}

Male Wistar rats, 6 to 8 weeks old, weighing 200-250 g, were supplied by the Animal Research Center at the First Clinical Medical School of Harbin Medical University, Harbin, China. The animals were maintained under standard conditions and were fed rodent chow and water. All surgical procedures and animal care were approved by the Institutional Ethics Committee.

Figure 1. Experimental protocol. Lipopolysaccharide (LPS, 0.5 $\mathrm{mg} / \mathrm{kg}$ body weight) was injected intravenously $24 \mathrm{~h}$ after $70 \%$ hepatectomy. Glycyrrhizin was administered at a dose of $20 \mathrm{mg} /$ $\mathrm{kg}$ body weight and samples were collected at the indicated times.

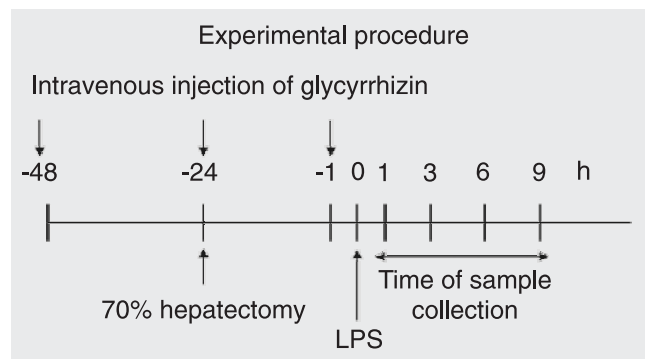

\section{Surgical procedures}

All surgical procedures were performed by one person in a standardized fashion. The glycyrrhizin injectable solution $(2 \mathrm{mg} / \mathrm{mL}$, $>95 \%$ purity, free of endotoxin) and lipopolysaccharide (LPS, O55:B5) were purchased from Shenzhen Jianan Pharmaceutic Ltd., Shenzhen, China, and Sigma-Aldrich Co., Shanghai, China, respectively. In preliminary experiments, we showed that the effect of $70 \%$ partial hepatectomy or LPS treatment alone on rat survival did not differ from the sham operation; thus, these two groups were excluded from the present study. The animals were randomly assigned to sham operation, saline-treated and glycyrrhizin-treated groups of 24 rats each. The rats in the glycyrrhizin-treated group received an injection of glycyrrhizin solution $(20 \mathrm{mg} /$ $\mathrm{kg}$ body weight) via the penile vein three times (at 48, 24, and $1 \mathrm{~h}$ prior to LPS injection; Figure 1). The rats in the saline-treated group received the same volume of physiological saline at the same times. After anesthesia with an intraperitoneal injection of $10 \%$ chloral hydrate $(300 \mathrm{mg} / \mathrm{kg}$ body weight), the animals underwent $70 \%$ hepatectomy (14). Twenty-four hours after surgery, LPS was injected into the rats via the penile vein at the dose of $0.5 \mathrm{mg} / \mathrm{kg}$ body weight (Figure 1). Animals in the sham operation group underwent laparotomy and the abdominal cavity was closed without partial hepatectomy or LPS injection. At each of the indicated times shown in Figure 1 (1, 3, 6, and $9 \mathrm{~h}$ after LPS injection), the rats (6 per group) were randomly sacrificed, and blood and liver samples were collected. Blood samples were centrifuged at $3000 \mathrm{~g}$ for 10 min to collect serum which was stored at $-80^{\circ} \mathrm{C}$.

\section{Biochemical assays}

The levels of aspartate aminotransferase (AST), alanine aminotransferase (ALT), and 
lactate dehydrogenase (LDH) in sera were measured with an automatic biochemical analyzer (Toshiba, Kawasaki, Japan).

\section{Histological examination}

Liver specimens were fixed in $10 \%$ buffered formalin, embedded in paraffin, stained with hematoxylin/eosin, and examined by light microscopy. The histopathological scoring analysis was performed blindly according to previously described methods (15). The result is reported as the sum of the individual scores from 0 (no findings) to 1 (mild), 2 (moderate), and 3 (severe) for each of the following six parameters: cytoplasmic color fading, vacuolization, nuclear condensation, nuclear fragmentation, nuclear fading, and erythrocyte stasis.

\section{TUNEL assay}

Serial 5 - $\mu \mathrm{m}$ thick liver sections were prepared. The terminal deoxynucleotidyl transferase-mediated dUTP nick end-labeling (TUNEL; Roche, Shanghai, China) staining of sections was performed according to manufacturer instructions, and examined by light microscopy. The apoptotic index was calculated as the percentage of stained cells as follows: apoptotic index $=$ number of apoptotic cells x $100 /$ total number of nucleated cells.

\section{Myeloperoxidase activity}

Myeloperoxidase (MPO) activity in liver tissue was determined by a method described in a report by our group (16).

\section{Immunohistochemical analysis}

Liver sections $(5 \mu \mathrm{m})$ were blocked with $2 \%$ BSA for $2 \mathrm{~h}$ and incubated overnight with antibodies against proliferating cell nuclear antigen (PCNA; Zhongshan Golden Bridge Biotechnology Co., Ltd., Beijing,
China), or rat NF-kB (Neo Markers Co., Fremont, CA, USA). They were subsequently incubated for $30 \mathrm{~min}$ with appropriate secondary antibodies using the PowerVision ${ }^{\mathrm{TM}}$ Two-step Histostaining Reagent (Zhongshan Golden Bridge Biotechnology Co.), and developed with Sigma FAST DAB (3,3'-diaminobenzidine tetrahydrochloride) and $\mathrm{CoCl}_{2} \mathrm{en}$ hancer tablets (Sigma-Aldrich). Sections were counterstained, mounted, and examined by microscopy. The number of PCNA-positive cells was counted in 30 random high-power fields, and the PCNA-labeling index was calculated according to the following formula: number of PCNA-positive cells x 100 / total number of cells. Similarly, the NF- $\kappa B-l a b e l-$ ing index was calculated by counting the cells with NF- $\kappa B$-positive nuclei.

\section{Western blotting}

The tissues were excised, minced and homogenized using a motor-driven homogenizer with (for detecting caspase-3) or without (for detecting cytochrome C) protein lysate buffer. Debris were removed by centrifugation at $10,000 \mathrm{~g}$ for $10 \mathrm{~min}$ at $4^{\circ} \mathrm{C}$. Without protein lysate buffer, the pellets contain the crude mitochondria fraction while the supernatants contain proteins from the cytoplasm, as described previously (17). The supernatants were resolved on $12 \%$ polyacrylamide SDS gels and electrophoretically transferred to polyvinylidene difluoride membranes. The membranes were blocked with $3 \%$ BSA overnight, and then incubated with primary antibodies against activated caspase3 or cytochrome $\mathrm{C}$ antibodies (Santa Cruz Biotechnology, Inc., Santa Cruz, CA, USA), and subsequently with alkaline phosphataseconjugated secondary antibody, and then developed with 5-bromo-4-chloro-3-indolyl phosphate/nitroblue tetrazolium (Tiangen Biotech Co. Ltd., Beijing, China). Blots were stained with an anti-tubulin antibody to confirm that each lane contained similar amounts of tumor homogenate. 


\section{ELISA}

TNF- $\alpha$ levels were measured with a TNF$\alpha$ ELISA kit (Jingmei Biotech Co. Ltd., Shenzhen, China) according to manufacturer instruction.

\section{Statistical analysis}

Survival rate data were analyzed statistically by the log rank test. All other data are reported as means \pm SEM and a least significant difference test was used to evaluate statistical significance. $\mathrm{P}<0.05$ was considered to be statistically significant.

\section{Results}

\section{Animal survival}

In a preliminary study, glycyrrhizin showed a beneficial survival effect on rats undergoing hepatectomy and LPS treatment. Sham-operated rats had a $100 \%$ survival rate. The rats submitted to $70 \%$ hepatectomy or LPS treatment alone survived during the monitoring period (data not shown). All animals undergoing $70 \%$ hepatectomy and LPS injection and treated with saline died within $36 \mathrm{~h}$ of LPS injection, and $30 \%$ of them (3/ 10) died as early as $12 \mathrm{~h}$ after LPS injection

Figure 2. Effect of glycyrrhizin on rat survival. A total of 30 rats were randomly assigned to three groups: glycyrrhizin, saline and sham. Glycyrrhizin or saline was pre-administered to the rats in the glycyrrhizin or saline groups, respectively as described in the legend to Figure 1, followed by $70 \%$ hepatectomy and lipopolysaccharide (LPS) injection. Rats in the sham group underwent laparotomy and the abdominal cavity was closed without partial hepatectomy or LPS injection. Data indicate percent animal survival (10 rats per group). The experimental protocol is given in Figure 1. PBS = phosphate-buffered saline.
(Figure 2). In contrast, pre-administration of glycyrrhizin significantly improved the survival rate of rats submitted to $70 \%$ hepatectomy and LPS injection $(\mathrm{P}<0.05)$, with the earliest deaths being observed 2 days after LPS treatment, and 50\% (5/10) of the rats

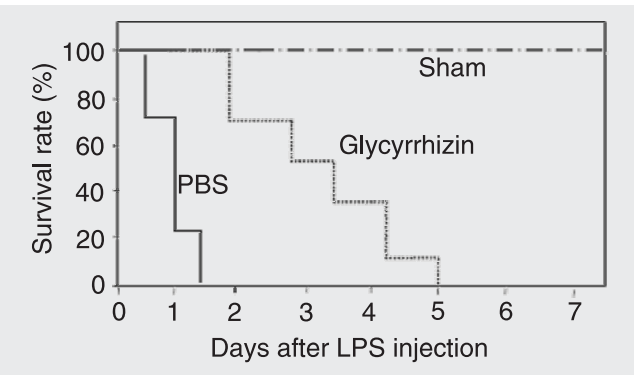

survived more than 3 days after LPS treatment (Figure 2).

\section{Liver function and histology}

Serum AST, ALT and LDH levels were analyzed to evaluate liver function. LPS significantly increased serum AST (Figure 3A), ALT (Figure 3B) and LDH (Figure 3C) levels in partially hepatectomized rats and these increases were significantly reduced by preadministration of glycyrrhizin. The results suggest that glycyrrhizin can attenuate the cellular damage that occurs as a result of hepatic damage by LPS after partial hepatectomy.

The serological changes were further confirmed by histological analysis. Histological alteration of the liver from hepatectomized rats treated with LPS was characterized by inflammatory cell infiltration, hemorrhagic change and focal necrosis in the midzone and periportal regions of the liver 6 $\mathrm{h}$ after LPS injection (Figure 4). In contrast, pre-treatment with glycyrrhizin markedly attenuated the pathological changes (Figure 4B). The liver injury scores for hepatectomized rats treated with LPS were significantly higher than those for sham-operated rats at all times $(\mathrm{P}<0.001$ in all cases $)$. Pretreatment with glycyrrhizin significantly decreased the histological scores compared with the saline-treated group $(\mathrm{P}<0.01$ in all cases; Figure 4C).

\section{Hepatocyte apoptosis}

LPS dramatically increased hepatocyte apoptosis in partially hepatectomized rats (Figure 5B) compared with sham-operated rats (Figure 5A), in which very few apoptotic cells were detected. Pre-treatment with glycyrrhizin markedly reduced the apoptosis of hepatocytes induced by LPS in partially hepatectomized rats (Figure 5C). The apoptotic index in the remnant livers from partially hepatectomized rats was signifi- 
cantly increased at the times indicated after LPS injection compared with sham-operated rats (Figure 5D). Glycyrrhizin significantly reduced the increases in the apoptotic index induced by LPS in remnant livers from hepatectomized rats by $62,51,50$, and $47 \%, 1,3,6$, and $9 \mathrm{~h}$ after LPS injection, respectively (Figure 5D).

We further investigated the molecular pathways involved in apoptosis by Western blot analysis of liver homogenates, which demonstrated that LPS up-regulated the expression of activated caspase- 3 and increased the expression of cytochrome $\mathrm{C}$ in the cellular cytoplasm of partially hepatectomized rats compared with sham-operated rats, and glycyrrhizin attenuated the up-regulation of caspase-3 and the release of cytochrome $\mathrm{C}$ into the cytoplasm induced by LPS (Figure $5 \mathrm{E})$.

\section{Inflammatory reaction}

LPS dramatically increased liver MPO activity in partially hepatectomized rats compared with sham-operated rats (Figure 6A) and this increase was significantly reduced by pre-treatment with glycyrrhizin. Serum TNF- $\alpha$ levels decreased in a time-dependent manner after LPS treatment in both salineand glycyrrhizin-treated rats, indicating that TNF- $\alpha$ plays a key role mainly in the early phase of liver injury induced by LPS in partially hepatectomized rats (Figure 6B).

We further investigated the effect of glycyrrhizin on the nuclear translocation of NF$\kappa \mathrm{B}$, the key upstream factor for various proinflammatory mediators. LPS led to marked overexpression of NF- $\kappa \mathrm{B}$ in the cell nuclei of partially hepatectomized rats (Figure 6D) compared with sham-operated rats (Figure 6C). However, pre-treatment with glycyrrhizin inhibited the expression of NF- $\mathrm{KB}$ in cell nuclei (Figure 6E). Quantitative data showed that LPS significantly increased the

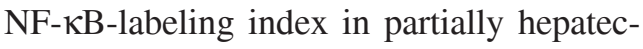
tomized rats compared with sham-operated rats $(\mathrm{P}<0.001$ in all cases $)$, while glycyrrhizin significantly reduced the NF- $\mathrm{KB}$-labeling index increased by LPS at the times indicated $(\mathrm{P}<0.01$ in all cases, Figure $6 \mathrm{~F})$

\section{Expression of proliferating cell nuclear antigen}

We investigated whether glycyrrhizin could improve the regeneration of remnant livers after partial hepatectomy by immunohistochemical analysis of PCNA expression. As shown in Figure 7A and B, pre-administration of glycyrrhizin increased the expression of PCNA in livers from partially hepatectomized rats treated with LPS compared with saline-treated rats. The PCNA-labeling index of hepatocytes was significantly in-

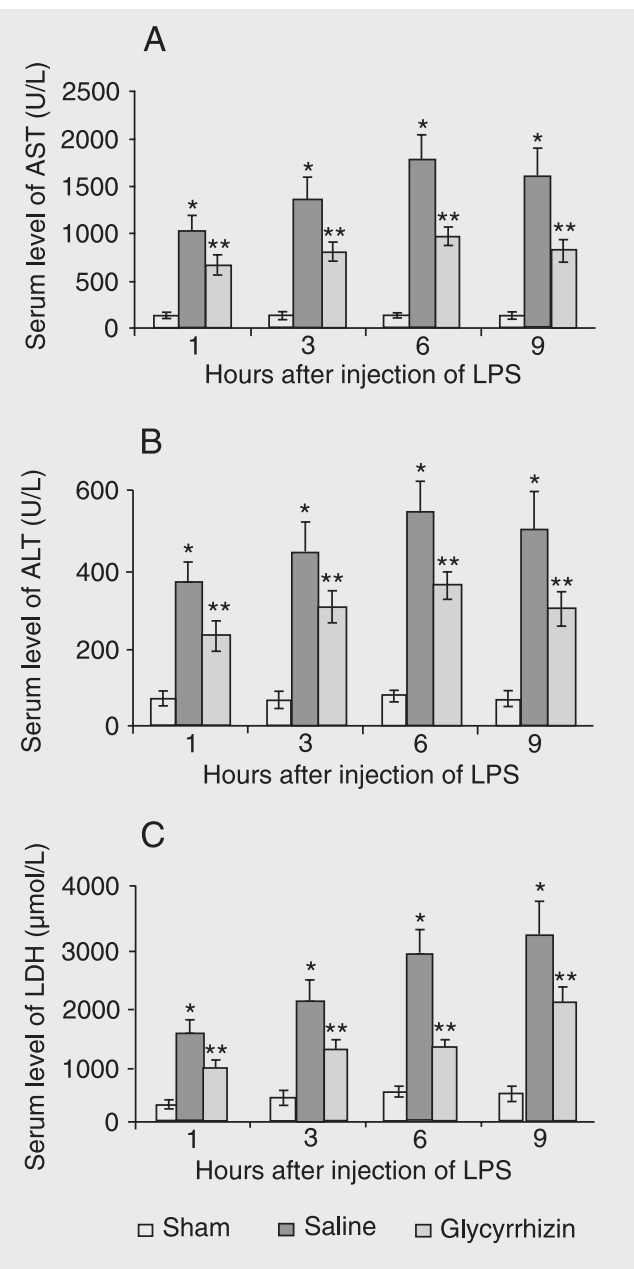

Figure 3. Serum levels of AST (A), ALT (B) and LDH (C). Blood samples were collected at the times indicated after LPS injection from partially hepatectomized rats, which were pretreated with glycyrrhizin or saline. Sham-operated rats served as control. AST = aspartate aminotransferase; $\mathrm{ALT}=$ alanine aminotransferase; $\mathrm{LDH}=$ lactate dehydrogenase; LPS = lipopolysaccharide. ${ }^{*} P<0.01$ for shamoperated rats compared to saline-treated rats; ${ }^{* *} \mathrm{P}<0.01$ for glycyrrhizin-treated rats compared to saline-treated rats (least significant difference test). The experimental protocol is given in Figure 1. 
Figure 4. Histological analysis of the liver. Representative photographs (400X magnification) of liver sections taken $6 \mathrm{~h}$ after injection of lipopolysaccharide (LPS) from partially hepatectomized rats, which were treated with saline (A) or glycyrrhizin (B). Arrows point to the necrotic hepatocytes. C, Histopathological scoring of hepatic injury performed as described in Material and Methods. ${ }^{*} P<0.001$ for sham-operated rats compared to saline-treated rats; ${ }^{* *} P<0.01$ for glycyrrhizin-treated rats compared to saline-treated rats (least significant difference test). The experimental protocol is given in Figure 1.
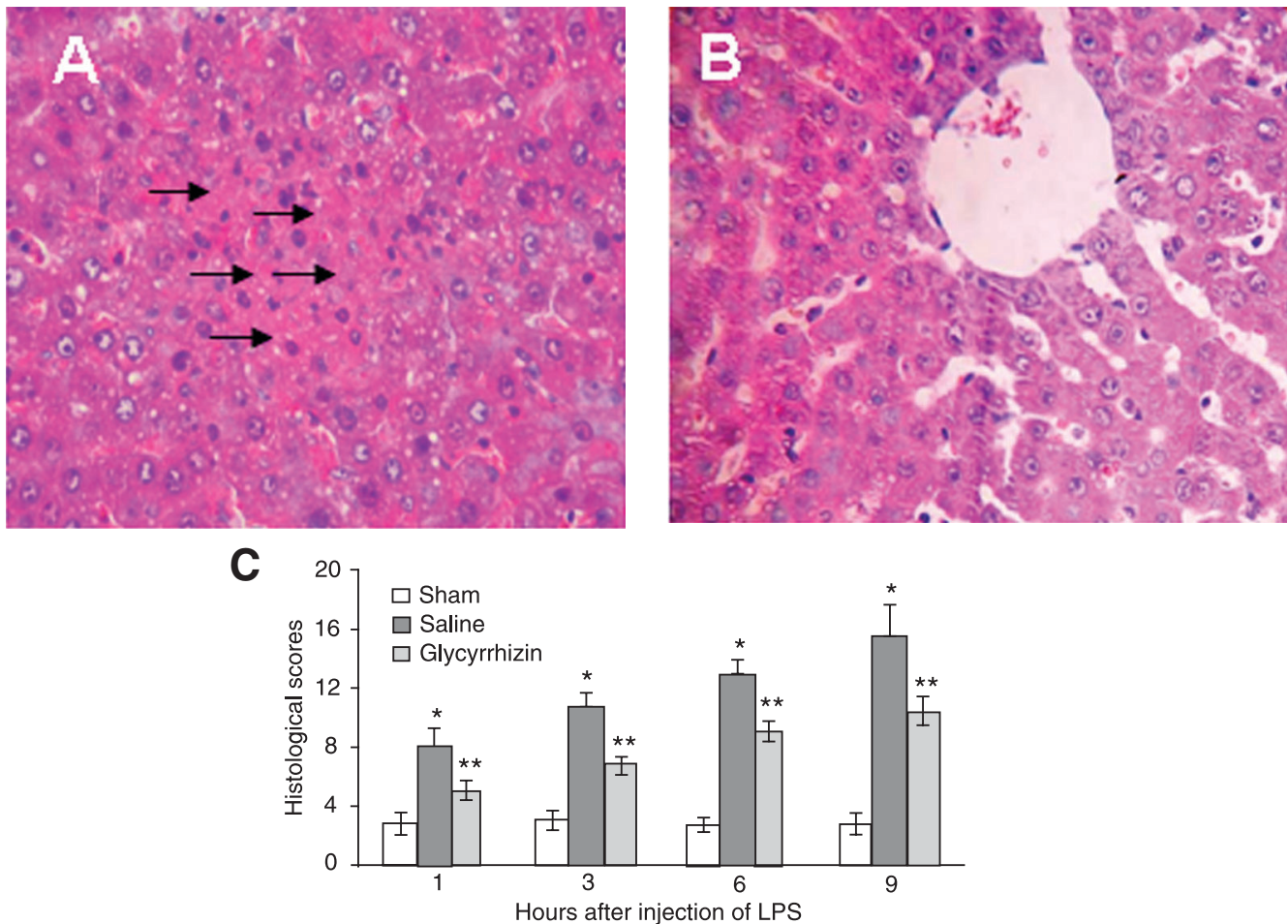

Figure 5. Anti-apoptotic effects of glycyrrhizin. Liver sections stained by TUNEL for apoptotic cells are illustrated. Representative photographs (400X magnification) of liver sections taken 6 $\mathrm{h}$ after injection of lipopolysaccharide (LPS) from partially hepatectomized rats, which were treated with saline (B) or glycyrrhizin (C). Sham-operated rats served as control (A). Arrows point to the TUNEL-positive cells. $D$, TUNEL-positive cells were counted to record the apoptotic index (\%). ${ }^{*} \mathrm{P}<0.01$ for saline-treated rats compared to sham-treated rats; ${ }^{* *} \mathrm{P}<0.01$ for glycyrrhizin-treated rats compared to saline-treated rats (least significant difference test). $E$, Western blot analysis of cytochrome $\mathrm{C}$ in the cellular cytoplasm and of caspase-3 in liver homogenates from sham-operated rats (lane 1), from hepatectomized rats treated with LPS and saline (lane 2), or glycyrrhizin (lane 3). The blot was reacted with an antibody against tubulin, which served as an internal control. The experimental protocol is given in Figure 1.
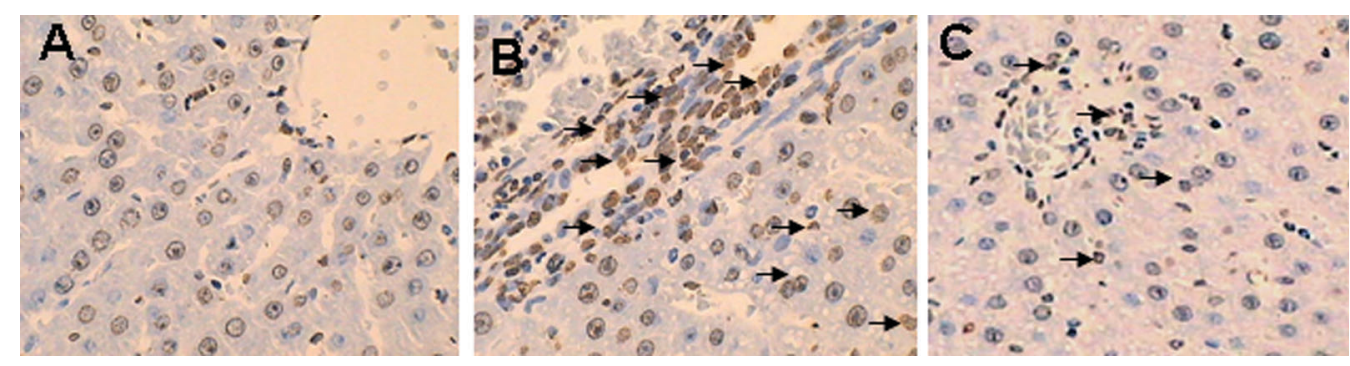

D

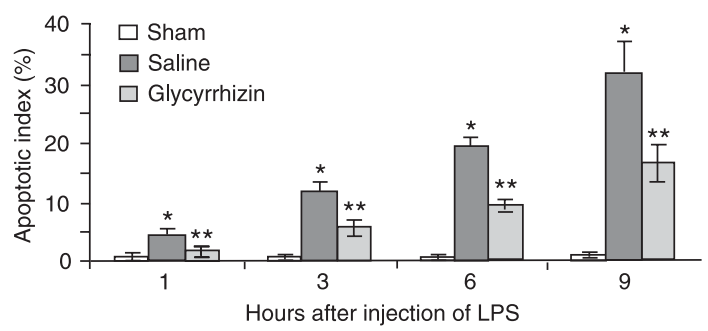

E

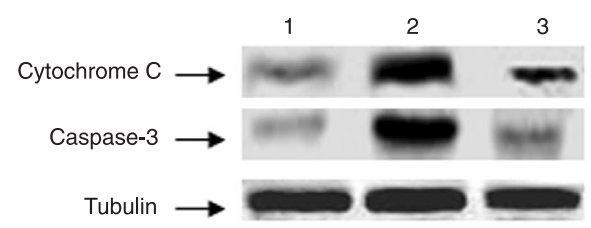



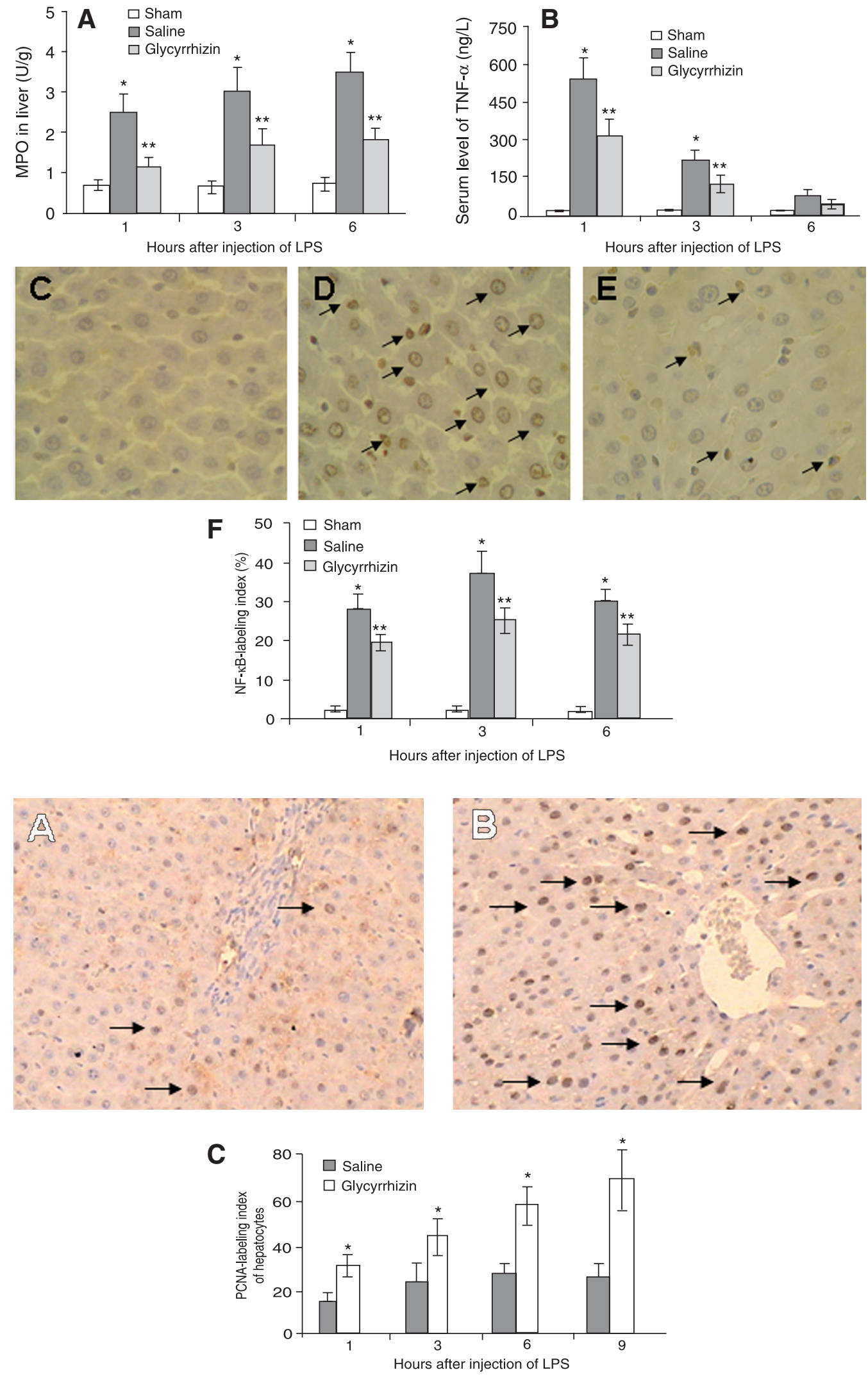

Figure 6. Glycyrrhizin inhibits the inflammatory reaction. At the indicated times after LPS injection, liver (for MPO) and serum (for TNF- $\alpha$ ) samples were collected from partially hepatectomized rats, which were pre-treated with saline or glycyrrhizin. Sham-operated rats served as control. The levels of MPO (A) and TNF$\alpha$ (B) were assessed as described in Material and Methods. $C-E$, Liver sections taken $6 \mathrm{~h}$ after injection of LPS from partially hepatectomized rats treated with saline (D) or glycyrrhizin (E), and sham-operated rats (C), immunostained for NF-kB expression (100X magnification). Arrows point to the NF-kB-positive cell nuclei. $F$, The cells with NF-kBpositive nuclei were counted to calculate the NF-kB-labeling index. LPS = lipopolysaccharide; MPO = myeloperoxidase; TNF- $\alpha$ = tumor necrosis factor-alpha; $\mathrm{NF}-\mathrm{\kappa B}=$ nuclear factor-kappa $\mathrm{B}$. ${ }^{*} P<0.001$ for saline-treated rats compared to sham-treated rats; ${ }^{* *} \mathrm{P}<0.01$ for glycyrrhizin-treated rats compared to saline-treated rats (least significant difference test). The experimental protocol is given in Figure 1.

Figure 7. Immunohistochemical analysis of proliferating cell nuclear antigen (PCNA). Liver sections taken $6 \mathrm{~h}$ after injection of lipopolysaccharide (LPS) from partially hepatectomized rats treated with saline (A) or glycyrrhizin (B), immunostained for PCNA expression (200X magnification) are illustrated. Arrows point to the PCNA-positive cells. $C$, PCNA-positive cells were counted to record the PCNA-labeling index for liver sections taken at the indicated times after LPS injection. ${ }^{*} P<0.01$ for glycyrrhizin-treated rats compared to saline-treated rats (least significant difference test). The experimental protocol is given in Figure 1. 
creased in glycyrrhizin-treated rats compared with saline-treated rats at the times indicated (Figure 7C).

\section{Discussion}

Seventy percent hepatectomy is not fatal in rats, but the cytotoxic sensitivity to endotoxin is increased during the early phase after hepatectomy (18). This increased sensitivity is partly associated with the reduced phagocytic function of the reticuloendothelial system after massive hepatectomy (19). Treatment with an even sublethal dose of LPS after hepatectomy induces liver failure (20). The present study demonstrated that the serum levels of AST, ALT, and LDH were significantly increased after LPS injection, and the liver injury was further sustained by pathological alterations including necrosis of the remnant liver in partially hepatectomized rats. Glycyrrhizin inhibited the increases of ALT, AST and LDH, and attenuated the morphological changes. These results indicate that glycyrrhizin prevents endotoxin-induced liver injury, and its protective effect against liver injury has been demonstrated previously in other experimental models $(10,12)$.

Hepatocyte apoptosis was also observed in livers damaged by LPS after partial hepatectomy, in agreement with a previous report (21). Apoptosis is initiated through the release of cytochrome from mitochondria. Cytochrome $\mathrm{C}$ binds to the CED-4 homologue Apaf-1, resulting in proteolytic processing and activation of pro-caspase-9. Active caspase- 9 then directly cleaves to and activates pro-caspase-3, initiating a cascade of additional caspase activation that culminates in apoptosis (22). The expression of activated caspase- 3 and the release of cytochrome $\mathrm{C}$ into the cytoplasm were both enhanced by LPS in the present study, and pre-treatment with glycyrrhizin down-regulated this change.

The role of glycyrrhizin in apoptosis is controversial since it has been reported that glycyrrhizin exhibits pro-apoptotic properties, but inhibits the mitochondrial permeability transition, reactive oxygen species generation and cytochrome $\mathrm{C}$ release at submicromolar concentration in the same study (23). This difference from the present study was possibly due to the different inducer of cytotoxicity and hepatocyte status in the cited study, in which hepatocyte apoptosis was induced by bile acid in vitro (23). On the other hand, apoptosis could also be induced by death domain receptor ligands such as TNF- $\alpha$ and Fas ligand (24). LPS binds to LPS-binding protein (LBP), and the LPS-LBP complex interacts with CD14 to form a ternary complex, LPS:LBP:CD14, which transfers LPS to the toll-like receptor 4 (TLR4) accessory protein MD2 complex, leading to activation of TLR4. Then, LPSactivated TLR4 activates NF- $\kappa B(25)$. Once activated, NF- $\mathrm{KB}$ is dissociated from its inhibitor, I- $\mathrm{\kappa B}$, and translocated into the nuclei, where it induces transcriptional up-regulation of various proinflammatory mediators such as TNF- $\alpha$ (26). Glycyrrhizin inhibits the translocation of NF- $\mathrm{KB}$ into the nuclei and reduces the levels of TNF- $\alpha$, thus inhibiting hepatocyte apoptosis via the death domain receptor pathway. Glycyrrhizin has already been demonstrated to attenuate antiFas antibody-induced liver injury (27).

During endotoxemia, inflammatory cytokines including TNF- $\alpha$ and neutrophil infiltration play important roles in liver injury (28). TNF- $\alpha$ is a key mediator of the cytokine cascade and tissue injury in sepsis (4), and is involved in the pathogenesis of LPS-induced liver injury (28). By producing oxidative stress, neutrophils activate Kupffer cells and contribute to microvascular dysfunction and edema formation (29). MPO, an enzyme present in neutrophils, is a widely used marker of neutrophil infiltration (30). The present study has demonstrated that LPS treatment increased MPO activity in the liver, suggesting that neutrophils contribute to the 
liver damage induced by LPS. Pre-treatment with glycyrrhizin decreases MPO activity, thus inhibiting neutrophil infiltration into the remnant liver and ameliorating the liver injury.

The proliferation of hepatocytes in the remnant liver determines the long-term survival of partially hepatectomized rats. In rats, after $70 \%$ hepatectomy, the mass restoration is complete within approximately 7 days and the rate of DNA synthesis in hepatocytes begins to increase after $12 \mathrm{~h}$ and reaches a peak at about $24 \mathrm{~h}$ after hepatectomy (31). In a previous report, all $70 \%$ hepatectomized rats died when they were treated with LPS (18). In the present study, LPS treatment retarded the regeneration of the remnant livers by down-regulating the expression of PCNA, a key marker of cell proliferation. However, pre-treatment with glycyrrhizin up-regulated the expression of PCNA, thus protecting the regeneration of livers harmed by LPS.

The present study demonstrated for the first time the protective activity of glycyrrhizin on endotoxin-induced acute liver injury after partial hepatectomy. Its protective mechanisms may be attributable to its antiinflammatory activity by inhibiting the release of proinflammatory mediators and neutrophil accumulation. Glycyrrhizin also exhibits anti-apoptotic activity through both the cytochrome and death domain receptor pathways, and pro-regenerative activity. The results indicate that glycyrrhizin may represent a potent drug for liver protection against endotoxin-induced injury, especially after massive hepatectomy.

\section{References}

1. Al-Hadeedi S, Choi TK, Wong J. Extended hepatectomy for hepatocellular carcinoma. Br J Surg 1990; 77: 1247-1250.

2. Matsumata T, Taketomi A, Kawahara N, Higashi H, Shirabe K, Takenaka K. Morbidity and mortality after hepatic resection in the modern era. Hepatogastroenterology 1995; 42: 456-460.

3. Kono H, Fujii H, Matsuda M, Yamamoto M, Matsumoto Y. Gadolinium chloride prevents mortality in hepatectomized rats given endotoxin. J Surg Res 2001; 96: 204-210.

4. Suzuki S, Nakamura S, Serizawa A, Sakaguchi T, Konno H, Muro H, et al. Role of Kupffer cells and the spleen in modulation of endotoxin-induced liver injury after partial hepatectomy. Hepatology 1996; 24: 219-225.

5. Seki S, Habu Y, Kawamura T, Takeda K, Dobashi H, Ohkawa T, et al. The liver as a crucial organ in the first line of host defense: the roles of Kupffer cells, natural killer (NK) cells and NK1.1 Ag+ T cells in T helper 1 immune responses. Immunol Rev 2000; 174: 35-46.

6. Deutschman CS, Haber BA, Andrejko K, Cressman DE, Harrison R, Elenko $\mathrm{E}$, et al. Increased expression of cytokine-induced neutrophil chemoattractant in septic rat liver. Am J Physiol 1996; 271: R593R600.

7. Bellezzo JM, Britton RS, Bacon BR, Fox ES. LPS-mediated NFkappa beta activation in rat Kupffer cells can be induced independently of CD14. Am J Physiol 1996; 270: G956-G961.

8. van Rossum TG, Vulto AG, Hop WC, Schalm SW. Chronic hepatitis $\mathrm{C}$ and portal hypertension: diagnosis, treatment with interferon, glycyrrhizin or propranolol. J Gastroenterol Hepatol 2001; 16: 789795.

9. Seeff LB, Lindsay KL, Bacon BR, Kresina TF, Hoofnagle JH. Complementary and alternative medicine in chronic liver disease. Hepatology 2001; 34: 595-603.
10. Yoshikawa M, Matsui Y, Kawamoto H, Umemoto N, Oku K, Koizumi $\mathrm{M}$, et al. Effects of glycyrrhizin on immune-mediated cytotoxicity. $J$ Gastroenterol Hepatol 1997; 12: 243-248.

11. Shiki Y, Shirai K, Saito Y, Yoshida S, Mori Y, Wakashin M. Effect of glycyrrhizin on lysis of hepatocyte membranes induced by anti-liver cell membrane antibody. J Gastroenterol Hepatol 1992; 7: 12-16.

12. Nagai T, Egashira T, Yamanaka $Y$, Kohno M. The protective effect of glycyrrhizin against injury of the liver caused by ischemia-reperfusion. Arch Environ Contam Toxicol 1991; 20: 432-436.

13. Kang DG, Sohn EJ, Mun YJ, Woo WH, Lee HS. Glycyrrhizin ameliorates renal function defects in the early-phase of ischemia-induced acute renal failure. Phytother Res 2003; 17: 947-951.

14. Higgins GM, Anderson RM. Experimental pathology of the liver. Arch Pathol 1931; 12: 186-202.

15. Heijnen BH, Straatsburg IH, Gouma DJ, Van Gulik TM. Decrease in core liver temperature with 10 degrees $\mathrm{C}$ by in situ hypothermic perfusion under total hepatic vascular exclusion reduces liver ischemia and reperfusion injury during partial hepatectomy in pigs. Surgery 2003; 134: 806-817.

16. Jiang H, Meng F, Li W, Tong L, Qiao H, Sun X. Splenectomy ameliorates acute multiple organ damage induced by liver warm ischemia reperfusion in rats. Surgery 2007; 141: 32-40.

17. Gao S, Chen J, Brodsky SV, Huang H, Adler S, Lee JH, et al. Docking of endothelial nitric oxide synthase (eNOS) to the mitochondrial outer membrane: a pentabasic amino acid sequence in the autoinhibitory domain of eNOS targets a proteinase K-cleavable peptide on the cytoplasmic face of mitochondria. J Biol Chem 2004; 279: 15968-15974.

18. Tsuji K, Kwon AH, Yoshida H, Qiu Z, Kaibori M, Okumura T, et al. Free radical scavenger (edaravone) prevents endotoxin-induced 
liver injury after partial hepatectomy in rats. J Hepatol 2005; 42: 94101.

19. Arii S, Shibagaki M, Takahashi S, Taki Y, Yokoo N, Jikko A, et al. Changes in the reticuloendothelial phagocytic function after partial hepatectomy. J Lab Clin Med 1985; 105: 668-672.

20. Mochida S, Ogata I, Hirata K, Ohta Y, Yamada S, Fujiwara K. Provocation of massive hepatic necrosis by endotoxin after partial hepatectomy in rats. Gastroenterology 1990; 99: 771-777.

21. Hase K, Xiong Q, Basnet P, Namba T, Kadota S. Inhibitory effect of tetrahydroswertianolin on tumor necrosis factor-alpha-dependent hepatic apoptosis in mice. Biochem Pharmacol 1999; 57: 14311437.

22. Li P, Nijhawan D, Budihardjo I, Srinivasula SM, Ahmad M, Alnemri ES, et al. Cytochrome $c$ and dATP-dependent formation of Apaf-1/ caspase-9 complex initiates an apoptotic protease cascade. Cell 1997; 91: 479-489.

23. Gumpricht E, Dahl R, Devereaux MW, Sokol RJ. Licorice compounds glycyrrhizin and 18beta-glycyrrhetinic acid are potent modulators of bile acid-induced cytotoxicity in rat hepatocytes. $J$ Biol Chem 2005; 280: 10556-10563.

24. Hatano E, Bradham CA, Stark A, limuro Y, Lemasters JJ, Brenner DA. The mitochondrial permeability transition augments Fas-induced apoptosis in mouse hepatocytes. J Biol Chem 2000; 275: 11814 11823.

25. Dauphinee SM, Karsan A. Lipopolysaccharide signaling in endothelial cells. Lab Invest 2006; 86: 9-22.

26. Su GL. Lipopolysaccharides in liver injury: molecular mechanisms of Kupffer cell activation. Am J Physiol Gastrointest Liver Physiol 2002; 283: G256-G265.

27. Okamoto T. The protective effect of glycyrrhizin on anti-Fas antibody-induced hepatitis in mice. Eur J Pharmacol 2000; 387: 229 232.

28. Sakaguchi T, Nakamura S, Suzuki S, Oda T, Ichiyama A, Baba S, et al. Participation of platelet-activating factor in the lipopolysaccharide-induced liver injury in partially hepatectomized rats. Hepatology 1999; 30: 959-967.

29. Jaeschke $H$. Reactive oxygen and mechanisms of inflammatory liver injury. J Gastroenterol Hepatol 2000; 15: 718-724.

30. Serafin A, Rosello-Catafau J, Prats N, Xaus C, Gelpi E, Peralta C. Ischemic preconditioning increases the tolerance of fatty liver to hepatic ischemia-reperfusion injury in the rat. Am J Pathol 2002; 161: $587-601$.

31. Michalopoulos GK, DeFrances MC. Liver regeneration. Science 1997; 276: 60-66. 